\title{
Utilization of Milk as an Oral Contrast Agent in CT Scan of the Abdomen
}

\author{
Asma Badawood', Nisreen Alsioufi², Samar Fathuddin'2, Nabeel Mishah ${ }^{2}$, Saddiq Jastaniah ${ }^{1}$ \\ ${ }^{1}$ Department of Diagnostic Radiology, Faculty of Applied Medical Sciences, King Abdulaziz University, Jeddah, \\ KSA \\ ${ }^{2}$ Radiology Department, King Abdulaziz University Hospital, Jeddah, KSA \\ Email: sjastaniah@kau.edu.sa
}

Received 29 April 2015; accepted 29 August 2015; published 1 September 2015

Copyright (C) 2015 by authors and Scientific Research Publishing Inc.

This work is licensed under the Creative Commons Attribution International License (CC BY). http://creativecommons.org/licenses/by/4.0/

(c) $\underset{\mathrm{EY}}{\mathrm{EP}}$ Open Access

\section{Abstract}

The present study aimed to evaluate using the whole milk as alternative oral contrast for the assessment of gastrointestinal CT enterography. Fifty patients undergo (18 female and 32 male) aged from 21 to 79 years (mean 45 years) undergo abdominal CT at the department of diagnostic radiology of King Abdulaziz University Hospital. They receive 1 - 1.5 liter whole milk. Siemens SOMATOM definition CT Scan Machine with 64 slices and dual source was utilized to image all patients in research population group where iodinated oral contrast media was replaced by a whole milk available in the local market. Patients were scanned as per the routine protocol used for CT Abdomen at KAUH-CT Unit: Helical Mode with 0.7 Pitch was performed on all paints in supine position using a 16-detector row scanner (Bright Speed S, General Electric-Milwaukee Wisconsin, USA). The following parameters were applied: collimation of $16 \times 0.625(1.25-3.75) \mathrm{mm}$, pitch of 3, Slice thickness: $5 \mathrm{~mm}$, gantry rotation time of $400 \mathrm{mAs}$, tube voltage of $120 \mathrm{KV}$ and scan time 11.05 second. The images of all cases were reviewed by two qualified radiologists. Conclusion: The present study suggested that whole milk was found to be an effective alternative negative contrast for the assessment of the jejunum, ileum and terminal ileum in CT enterography. It was excellent for gastric imaging and we recommended replacing milk as negative oral contrast since it is available and cost effective. Further research to be conducted with modified milk drinking timing for better large bowel distention.

\section{Keywords}

CT, Contrast Media, Milk, Abdomen, Bowl, Stomach

\section{Introduction}

It is essential in CT scanning of abdomen to fill the stomach, small bowl, large bowl and the rectum by a con-

How to cite this paper: Badawood, A., Alsioufi, N., Fathuddin, S., Mishah, N. and Jastaniah, S. (2015) Utilization of Milk as an Oral Contrast Agent in CT Scan of the Abdomen. Advances in Computed Tomography, 4, 33-41.

http://dx.doi.org/10.4236/act.2015.43005 
trast media (oral and rectal) in order to enable us to distinguish it from adjacent anatomical structure such as liver, pancreas, lymph nodes and pathological structures. Iodinated oral and rectal contrasts are used all over the world to visualize gastrointestinal tract and reach optimal distension to discriminate different organs and lymph nodes from the bowl. Replacement of oral iodinated contrast media by milk can help us to reduce the cost, improve patient care and patient safety in CT scan with better tolerance of milk compared to the oral contrast [1] [2].

Higher attenuated oral contrast agents in the form of iodinated compounds or barium suspensions have been used effectively for computed tomographic (CT) examinations of the abdomen [3] [4]. Few drawbacks and unpleasant taste may create difficulties in interpretation, such as formation of pseudotumor due to improper mixing of contrast material with the contents of the bowel and poor mural discrimination [5].

Neutral contrast agents leads distension of the stomach with water either by drinking or through a nasogastric tube, allows better visualization of gastric wall thickness, Water is little used in the small bowel: a water-filled loop of bowel can have a similar appearance to that of a cyst or abscess [6]-[8].

Negative contrast agents are quite often residual gas within the bowel serves as a marker. This is especially true in the colon. If a nasogastric tube is in place, air can be injected into the stomach and small bowel. With some of the earlier scanners, excessive air led to streak artifacts: these are no longer a problem with most of the modern scanners. With significant amounts of gas, window settings wider than usual are of help. A number of products containing fat, such as mineral oil, have been used. Currently, these have a limited application [7].

Positive contrast agents like iodinated water-soluble agents and various barium sulfate suspensions have been used. The ideal such contrast agent should lead to ready bowel differentiation from surrounding structures without any resultant artifacts Full-strength suspensions as used in conventional radiography obviously have no role. The resultant high contrast simply leads to streak artifacts [9]-[11].

A water-soluble agents: Dilute solutions of the ionic and nonionic water-soluble contrast agents have been used perorally.

Replacement of oral iodinated contrast media by milk can help us to reduce the cost, improve patient care and patient safety in CT scan with better tolerance of milk compared to the oral contras so the aim of the current research study was to evaluate the cost effectiveness and assessment of milk as an oral contrast-enhanced abdominal CT that replacing oral iodinated contrast media.

\section{Ease of Use}

\subsection{Material and Methods}

Fifty patients undergo whole abdominal CT at the department of diagnostic radiology of King Abdulaziz University Hospital, were assigned to receive $1000 \mathrm{ml}-1.5$ liter whole milk as oral contrast agent after obtaining research ethical approval; a prospective study was developed. The study group included 18 female and 32 male, ranging in age from 21 to 79 Years with the mean age is 45 years. Inclusion criteria were the following: patients above 18 years of age with spontaneous requests for abdomen and pelvis CT studies with indication for utilization of intravenous and oral contrast agents. Exclusion criteria were the following: history of gastrointestinal tract surgery, known neoplasia of the gastrointestinal tract, intolerance to lactose, current intake of laxatives, and presence of gastrointestinal symptoms such as nausea, vomiting and diarrhea; besides pregnancy.

The images were independently interpreted by two radiologists who were blinded to the contrast agents used with four- and ten-year experience in abdominal CT and one of them blind to the type of oral contrast agent utilized in the study. The degree of bowel distension was qualitatively scored on a four-point scale Poor, Fair, Good, and Excellent.

\subsection{Patient Safety and Preparation}

To exclude any possibility of reactions or unpleasant feeling after milk intake of such large volumes i.e. 1000 $1500 \mathrm{cc}$, we asked the patient of any previous allergic reactions from milk or dairy products any patients who have any drought about this issue is excluded from our study.

The patients are fastened 6 hours to prevent the side effect of contrast material. The patient was placed supine or prone on the imaging table. The patients are instructed to hold breath after full expiration. Siemens SOMATOM definition CT Scan Machine with 64 slices and dual source was utilized to image all patients in re- 
search population group where iodinated oral contrast media was replaced by a whole milk available in the local market. Patient asked to drink from one liter to one and a half liter of whole milk (6 cups 1 Liter each) one hour before the examination. Patients were scanned as per the routine protocol used for CT Abdomen at KAUH-CT Unit: Helical Mode with 0.7 Pitch was performed on all paints in supine position using a 16-detector row scanner (Bright Speed S, General Electric-Milwaukee Wisconsin, USA). The following parameters were applied: collimation of $16 \times 0.625(1.25-3.75) \mathrm{mm}$, pitch of 3, Slice thickness: $5 \mathrm{~mm}$, gantry rotation time of $400 \mathrm{mAs}$, tube voltage of $120 \mathrm{Kv}$ and scan time 11.05 second.

\subsection{Protocol Used for Our Study}

The images of all cases were reviewed by two qualified radiologists, the first radiologist knows about the replacement of iodinated oral contrast by whole milk while the second radiologist was blind. Both were asked to review the images then to fill the evaluation form designed to evaluate the distension of hallow organs in the digestive system mainly to visualize distension of stomach, jejunum, ileum and proximal large bowel. The answers were categorized as per the degree of successful distention of each part and the answers of each radiologist were calculated for each category per part.

The analysis of evaluation distension of gut was dependent on the CT images. This was done to assess the usefulness of the contrast visualization of the gut effectively for better evaluation of gastrointestinal tract that can help us to reduce the cost, improve patient care and patient safety in CT scan with better tolerance of milk compared to the oral contrast.

\section{Results}

The evaluation forms was collected from the two radiologists involved in this research to evaluate using the whole milk in CT Imaging of digestive system as alternative oral contrast as shown in Figure 1 and Figure 2 and Table 1. Gastric distention of milk was appreciated by the $1^{\text {st }}$ radiologist as excellent distention in 26 cases out of 50 total cases representing 52\% while the $2^{\text {nd }}$ radiologist scored Excellent for 33 cases representing $66 \%$. The average excellent score by both radiologist was $59 \%$. As for the Good distention the $1^{\text {st }}$ radiologist scored 20 cases out of 50 cases representing $40 \%$ while the $2^{\text {nd }}$ radiologist scored good for 14 cases representing $28 \%$. The average score for Good by both radiologists was $34 \%$.
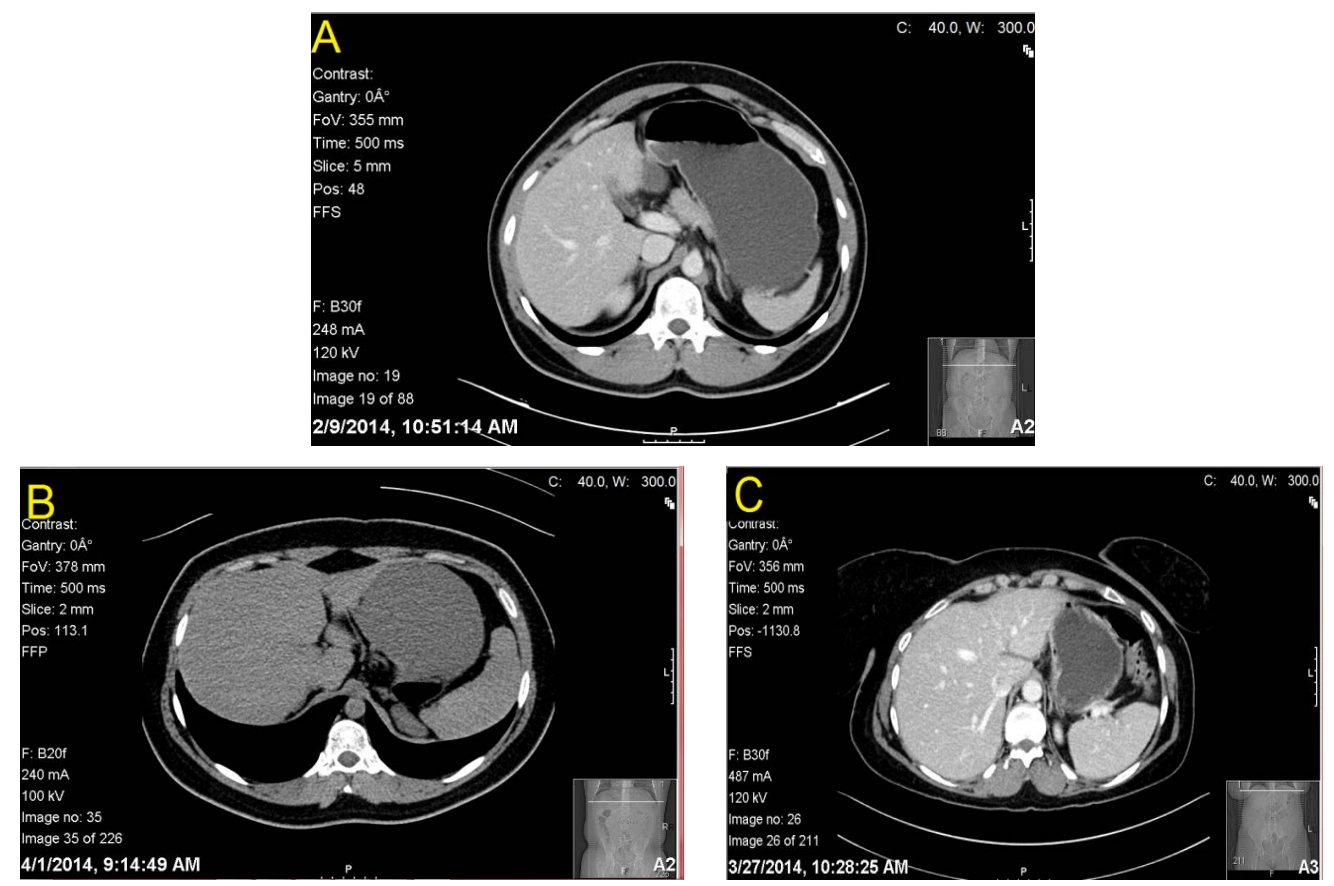

Figure 1. Gastric distention was demonstrated with excellent appearance (A), very good appearance (B) and fair appearance (C). 


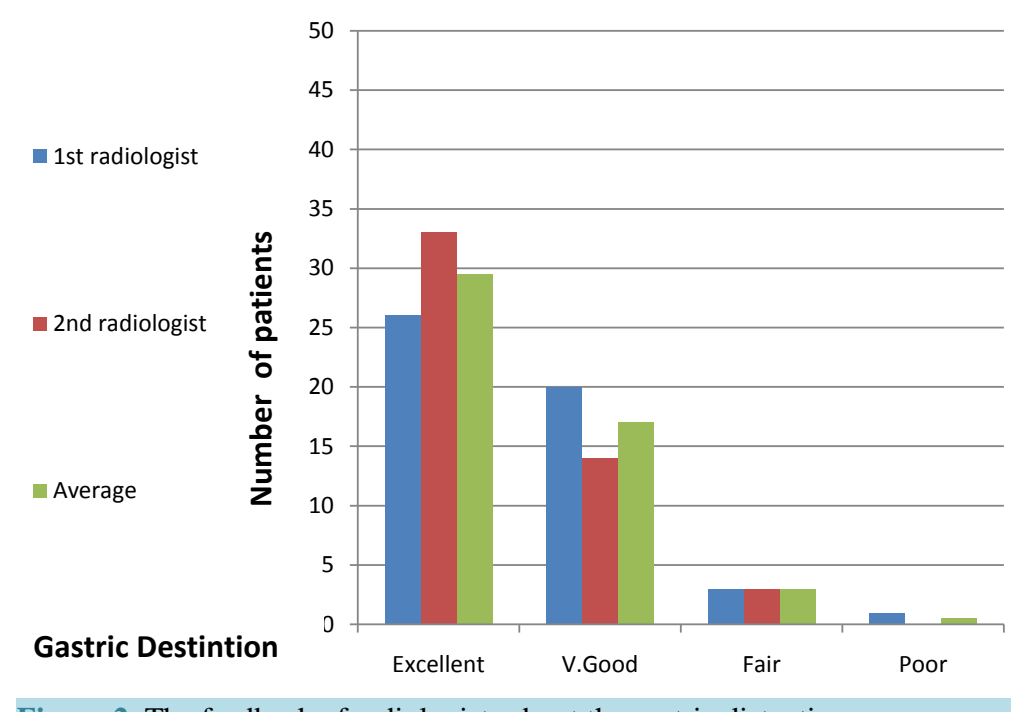

Figure 2. The feedback of radiologists about the gastric distention.

Table 1. Gastric distention.

\begin{tabular}{ccccc}
\hline & Excellent & Good & Fair & Poor \\
\hline $1^{\text {st }}$ radiologist & 26 & 20 & 3 & 1 \\
$2^{\text {nd }}$ radiologist & 33 & 14 & 3 & 0 \\
Average & 29.5 & 17 & 3 & 5 \\
\hline
\end{tabular}

The Fair distention was 3 cases out of 50 cases representing $6 \%$ for the $1^{\text {st }}$ radiologist, also, the $2^{\text {nd }}$ radiologist scored Fair for 3 cases representing 6\%. Accordingly, the average score for fair by both radiologists was 6\%. Poor distention was selected for 1 case out of 50 cases representing $2 \%$ by the $1^{\text {st }}$ radiologist, while the $2^{\text {nd }}$ radiologist scored poor for none of the cases representing $0 \%$. The average score for Poor by both radiologists was $1 \%$. Gastric distention was demonstrated with excellent appearance, very good appearance and fair appearance.

Duodenum distention of milk was appreciated by the $1^{\text {st }}$ radiologist as excellent distention in 5 cases out of 50 total cases representing $10 \%$ while the $2^{\text {nd }}$ radiologist scored Excellent for 1 case representing $2 \%$. The average excellent score by both radiologist was $6 \%$. As for very good distention the $1^{\text {st }}$ radiologist scored 21 cases out of 50 cases representing $42 \%$ while the $2^{\text {nd }}$ radiologist scored very good for 7 cases representing $14 \%$. The average score for Good by both radiologists was $28 \%$. The Fair distention was 23 cases out of 50 cases representing $46 \%$ for the $1^{\text {st }}$ radiologist, while the $2^{\text {nd }}$ radiologist scored Fair for 16 cases representing 32\%. The average score for Fair by both radiologists was 39\%. Poor distention in was selected for 1 case out of 50 cases representing $2 \%$ by the $1^{\text {st }}$ radiologist, while the $2^{\text {nd }}$ radiologist scored Poor for 26 representing $52 \%$. The average score for Poor by both radiologists was 27\% (Table 2 and Figure 3 and Figure 4).

Jejunum distention of milk was appreciated by the $1^{\text {st }}$ radiologist as excellent distention in 1 case out of 50 total cases representing $2 \%$ also the $2^{\text {nd }}$ radiologist scored Excellent for 1 case representing $2 \%$. Accordingly the average excellent score by both radiologist was $2 \%$. As for $\mathrm{V}$. Good distention the $1^{\text {st }}$ radiologist scored 8 cases out of 50 cases representing $16 \%$ while the $2^{\text {nd }}$ radiologist scored V. Good for 9 cases representing $18 \%$. The average score for V. Good by both radiologists was $17 \%$. The fair distention was 21 cases out of 50 cases representing $42 \%$ for the $1^{\text {st }}$ radiologist, while the $2^{\text {nd }}$ radiologist scored fair for 29 cases representing $58 \%$. The average score for Fair by both radiologists was 50\%.Poor distention was selected for 20 cases out of 50 cases representing $40 \%$ by the $1^{\text {st }}$ radiologist while the $2^{\text {nd }}$ radiologist scored Poor for 11 cases representing $22 \%$. The average score for poor by both radiologists was 31\% (Table 3 and Figure 5 and Figure 6).

Ilium distention of milk was appreciated by the $1^{\text {st }}$ radiologist as Excellent distention in 1 case out of 50 total cases representing $2 \%$ While the $2^{\text {nd }}$ radiologist scored Excellent for none of the cases representing $0 \%$. The average Excellent score by both radiologist was $1 \%$. As for V. Good distention the $1^{\text {st }}$ radiologist scored 7 cases out of 50 cases representing $14 \%$ while the $2^{\text {nd }}$ radiologist scored V. Good for 8 cases representing $16 \%$. The 

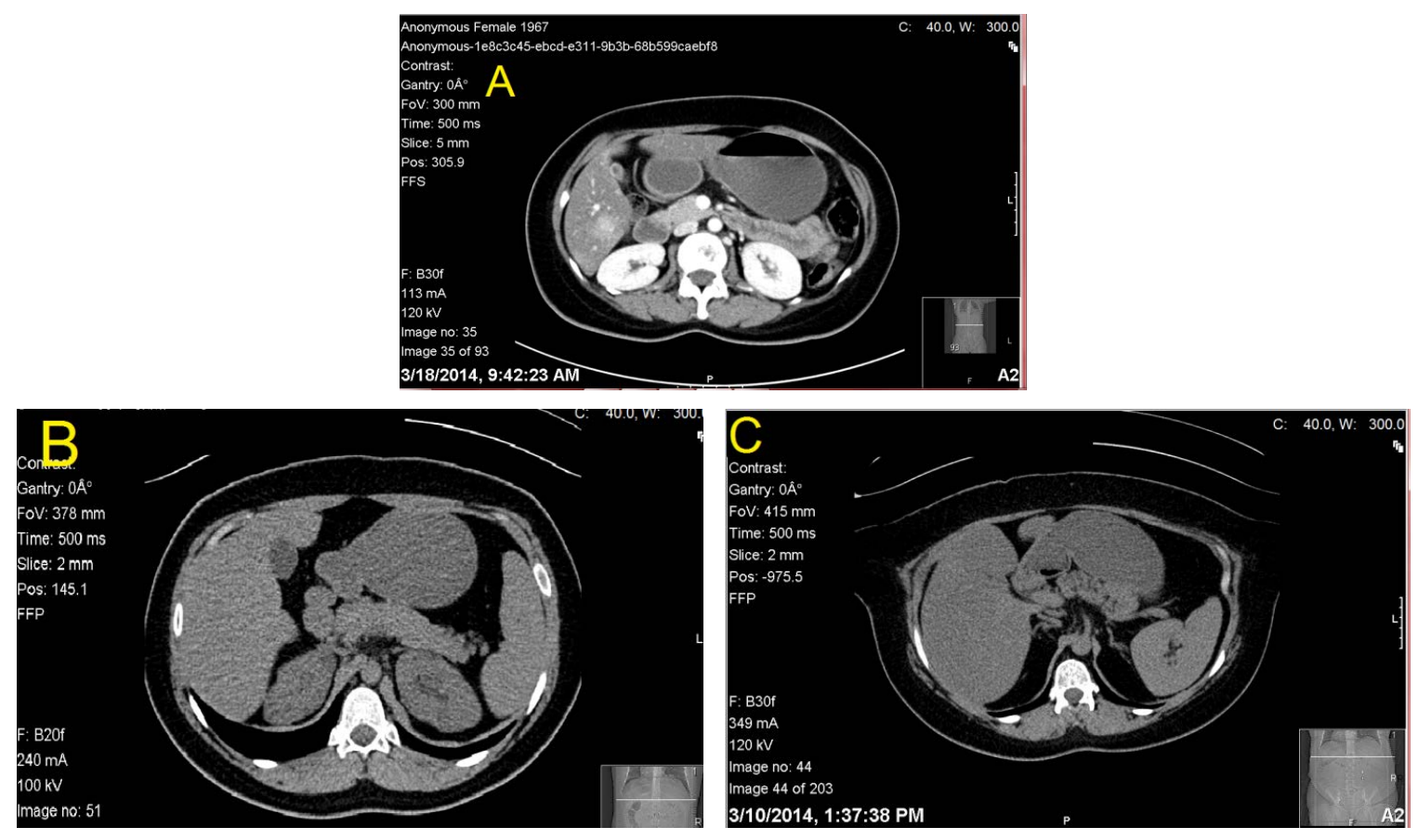

Figure 3. Showing the duodenum distention was demonstrated with very good appearance (A) fair appearance (B) and poor appearance $(\mathrm{C})$.

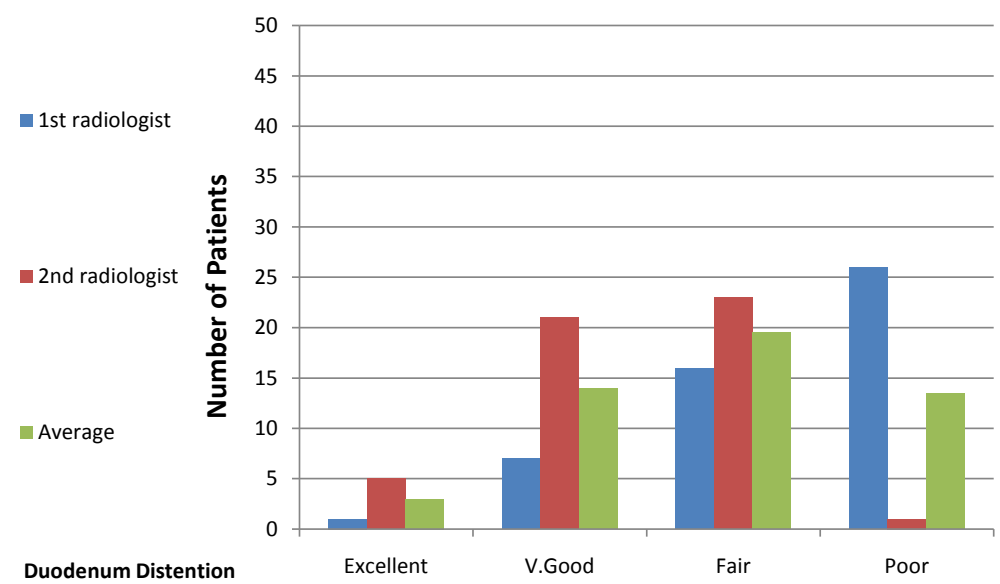

Figure 4. The feedback of radiologists about the duodenum distention.

Table 2. Duodenum distention.

\begin{tabular}{ccccc}
\hline & Excellent & Good & Fair & Poor \\
\hline $1^{\text {st }}$ Radiologist & 5 & 21 & 23 & 1 \\
$2^{\text {nd }}$ Radiologist & 1 & 7 & 16 & 26 \\
Average & 3 & 14 & 19.5 & 13.5 \\
\hline
\end{tabular}

Table 3. Jejunum distention.

\begin{tabular}{ccccc}
\hline & Excellent & Good & Fair & Poor \\
\hline $1^{\text {st }}$ Radiologist & 1 & 8 & 21 & 20 \\
$2^{\text {nd }}$ Radiologist & 1 & 9 & 29 & 11 \\
Average & 1 & 8.5 & 25 & 15.5 \\
\hline
\end{tabular}



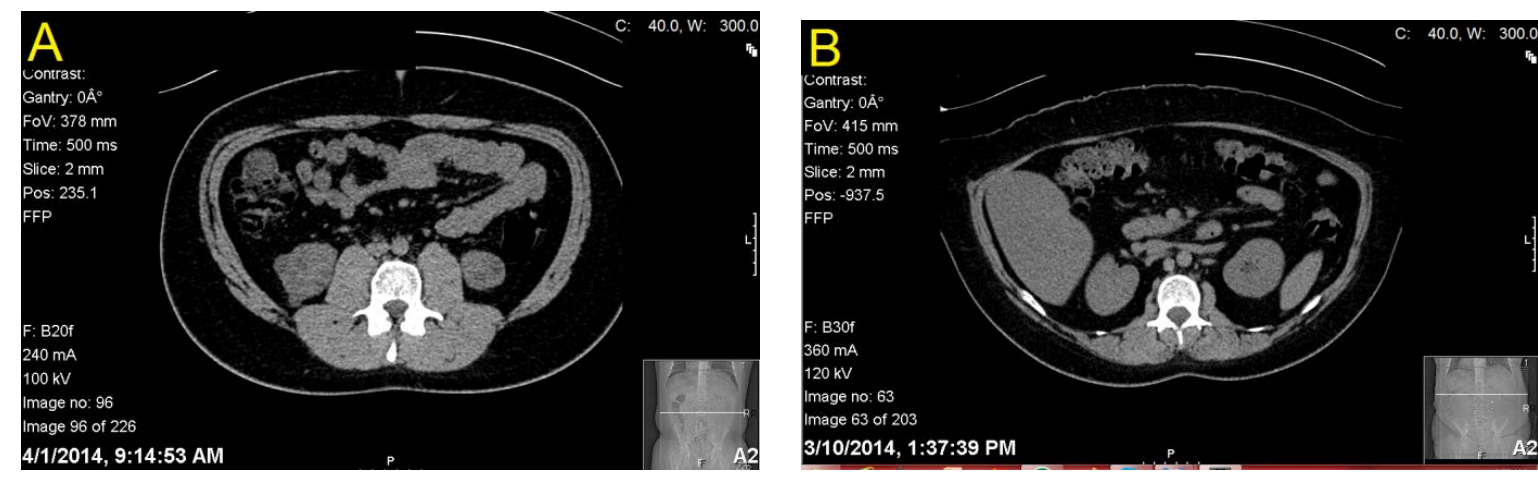

Figure 5. (A) Jejunum distention was demonstrated with fair appearance; (B) Jejunum distention was demonstrated with poor appearance.

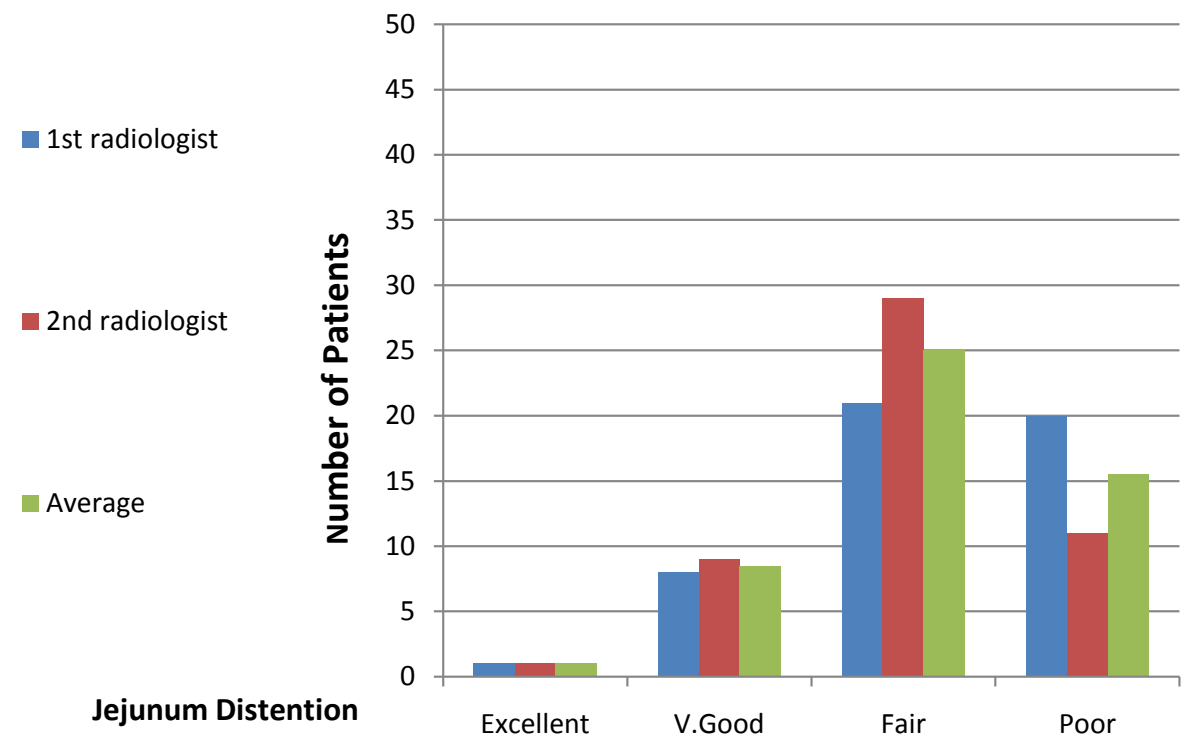

Figure 6. The feedback of radiologists about the Jejunum distention.

average score for V. Good by both radiologists was $15 \%$. The Fair distention was 18 cases out of 50 cases representing $36 \%$ for the $1^{\text {st }}$ radiologist, while the $2^{\text {nd }}$ radiologist scored Fair for 15 cases representing $30 \%$. The average score for Fair by both radiologists was 33\%.Poor distention was selected for 24 cases out of 50 cases representing $48 \%$ by the $1^{\text {st }}$ radiologist, while the $2^{\text {nd }}$ radiologist scored Poor for 27 cases representing $54 \%$. The average score for Poor by both radiologists was 51\% (Table 4 and Figure 7 and Figure 8).

\section{Discussions}

Negative oral contrast agents used for evaluation of lesions of the gut which could be better delineated due to gut distension. Volumen with $0.1 \%$ barium sulphate suspension is being recommended as low-density contrast agent. However, there are some drawbacks such as cost, limited availability, and some patient discomfort such as nausea and vomiting as well as poor palatability [12] [13].

Low-attenuation oral contrast agent using water for helical CT of the abdomen was used by Winter [14] which exquisite luminal and upper gastrointestinal tract mucosal depiction. However, using of water requires the adding of a smooth muscle relaxant of the upper gastrointestinal and does not give adequate evaluation of the distal small bowel [15].

We expected that used milk of $4 \%$ fat as contrast agent used orally could produce results comparable to those obtained with corn oil emulsion but without the unfit high content of fat. Furthermore, the milk fat could be sufficient to decrease peristalsis of gastrointestinal tract. This could allow upper gastrointestinal tract distention and mural discrimination that were superior to those obtained by water [16]. 

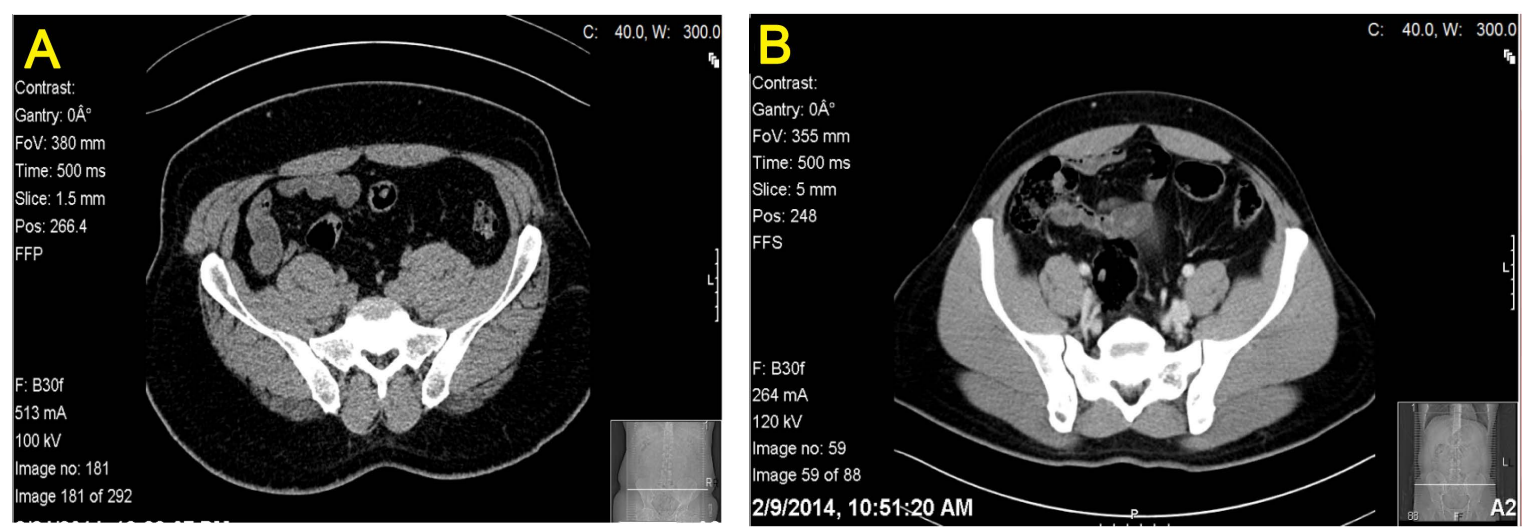

Figure 7. Ilium distention was demonstrated with fair appearance (A) and poor appearance (B).

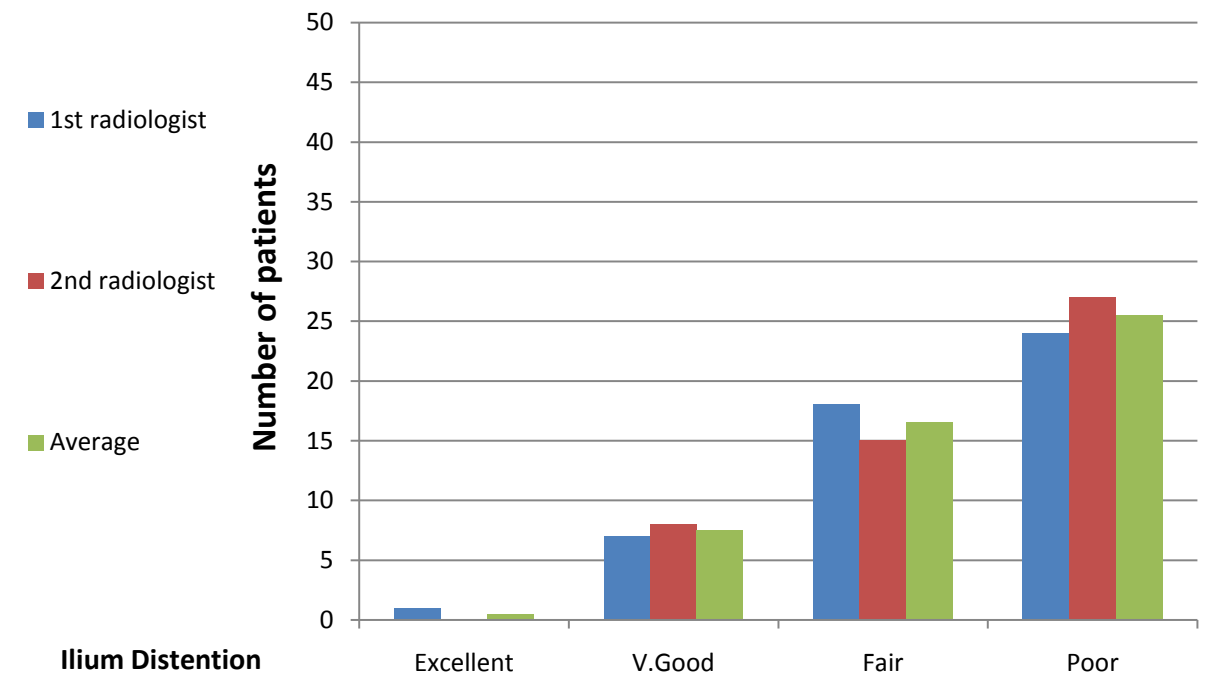

Figure 8. The feedback of radiologists about the Ilium distention.

Table 4. Ilium distention.

\begin{tabular}{ccccc}
\hline & Excellent & Good & Fair & Poor \\
\hline $1^{\text {st }}$ Radiologist & 1 & 7 & 18 & 24 \\
$2^{\text {nd }}$ Radiologist & 0 & 8 & 15 & 27 \\
Average & 0.5 & 7.5 & 16.5 & 25.5
\end{tabular}

Although promising results have been achieved, but the study sample was limited to those patients undergoing CT Scan of Abdomen and Pelvis with the use of water as oral contrast. Additionally, the degree of distention evaluated by both radiologists was subjective depending on naked eye. The timing of drinking milk was not properly adjusted to allow for filling the large bowel due to pre scheduled appointments [17].

Hence, the replacement with whole milk with $4 \%$ as in the present study, fat content has been shown to be as effective in distension of the gut and also takes care of the disadvantages of Volumen. It is also a preferred agent in pediatric cases. A larger study needs to further corroborate these observations. Gastric distension and visualization as well as duodenum, jejunum, and proximal bowel was significantly improved with milk as a low-attenuation contrast agent [12] [18].

Gastric distention of milk was appreciated where the present study demonstrated excellent distension and visualization of the stomach and was good and fair for duodenum, jejunum was significantly improved with milk as a contrast agent. It showed an attractive oral contrast agent, giving good gastrointestinal distension and land- 
mark discrimination. These results are in agreement with that Mitka 2007 [19] and Meyer and Gawde 2012 [12] while A pisarnthanarak et al. (2013) [20] stated that apart from collapse of the gallbladder, increase of GI adverse symptoms was another major drawback of whole milk when used as CT oral contrast, especially in Thai people [21].

Results of Thompson [22] demonstrated that whole milk were superior to other agents used for gastrointestinal distention, mural visualization, and pancreas-duodenum discrimination. In bowel loop discrimination, results with 3.8\% milk were synonymous to those with barium but superior to water for bowel distension and significantly superior to $0.1 \%$ gastrografin. However, $10 \%$ of patients who received $3.8 \%$ milk reported immediate post-test diarrhoea. No side effects were documented for patients who received water and $0.1 \%$ gastrografin. $3.8 \%$ milk is an effective and superior neutral oral contrast agent for the assessment of the jejunum, ileum and terminal ileum in CT enterography [23]. However, further studies are needed to explore other suitable oral contrast agents for CT enterography in lactose- or cow's milk-intolerant patients [24].

\section{Conclusion}

The present study indicated that whole milk was found to be an effective alternative negative contrast for the assessment of the jejunum, ileum and terminal ileum in CT enterography. It was excellent for gastric imaging and we recommended replacing milk as negative oral contrast since it is available and cost effective. Further research is to be conducted with modified milk drinking timing for better large bowel distention.

\section{References}

[1] Dizendorf, E.V., Treyer, V., Von Schulthess, G.K. and Hany, T.F. (2002) Application of Oral Contrast Media in Coregistered Positron Emission Tomography-CT. American Journal of Roentgenology, 179, 477-481. http://dx.doi.org/10.2214/ajr.179.2.1790477

[2] Megibow, A.J., Babb, J.S., Hecht, E.M., Cho, J.J., Houston, C. and Boruch, M.M. (2006) Evaluation of Bowel Distention and Bowel Wall Appearance by Using Neutral Oral Contrast Agent for Multidetector Row CT. Radiology, 236, 87-95. http://dx.doi.org/10.1148/radiol.2381041985

[3] Nyman, U., Dinnetz, G. and Anderson, I. (1984) E-Z-CAT: An Oral Contrast Medium for Use in Computed Tomography of the Abdomen. Acta Radiologica, 25, 121-124. http://dx.doi.org/10.1177/028418518402500206

[4] Raptopoulos, V. (1989) Technical Principles in CT Evaluation of the Gut. Radiologic Clinics of North America, 27, 631-651.

[5] Fisher, J.K. (1982) Normal Colon Wall Thickness on CT. Radiology, 145, 415-418. http://dx.doi.org/10.1148/radiology.145.2.7134445

[6] Nakada, K., Brown, R.S., Fisher, S.J., Higashi, T., Walter, G.L., Wahl, R.L., et al. (2001) Histological Localization of Tritiated FDG in the GI Tract: An Autoradiographic Study (abstr). Journal of Nuclear Medicine, 42, 25.

[7] Antoch, G., Kuehl, H., Kanja, J., Lauenstein, T.C., Schneemann, H., Hauth, E., et al. (2004) Dual-Modality PET/CT Scanning with Negative Oral Contrast Agent to Avoid Artifacts: Introduction and Evaluation. Radiology, 230, 879-885. http://dx.doi.org/10.1148/radiol.2303021287

[8] Makarawo, T.P., Negussie, E., Malde, S., Tilak, J., Gayagoy, J., Watson, J., Francis, F., Lincoln, D. and Jacobs, M.J. (2013) Water as a Contrast Medium: A Re-Evaluation Using the Multidetector-Row Computed Tomography. The American Surgeon, 79, 728-733.

[9] Sun, X.G., Huang, G., Zhang, C., Chen, Y., Xue, H., Zheng, P., et al. (2006) Comparison of the Effect of Positive and Negative Oral Contrast Agent in FDG PET/CT Scan. Journal of Nuclear Medicine, 47, 149.

[10] Bae, K.T. (2010) Intravenous Contrast Medium Administration and Scan Timing at CT: Considerations and Approaches. Radiology, 256, 32-61. http://dx.doi.org/10.1148/radiol.10090908

[11] Oliva, M.R., Erturk, S.M., Ichikawa, T., Rocha, T., Ros, P.R., Silverman, S.G. and Mortele, K.J. (2012) Gastrointestinal Tract Wall Visualization and Distention during Abdominal and Pelvic Multidetector CT with a Neutral Barium Sulphate Suspension: Comparison with Positive Barium Sulphate Suspension and with Water. JBR-BTR, 95, $237-242$.

[12] Meyer, S.A. and Gawde, S. (2012) Utility of Negative Oral Contrast (Milk with 4\% Fat) in PET-CT Studies. Indian Journal of Nuclear Medicine, 27, 151-155.

[13] Rupa, S.A., Al Azad, S. and Chakraborty, N.R. (2013) Milk-An Effective Low-Attenuation Computed Tomography Oral Contrast Medium. Journal of Enam Medical College, 3, 71-76.

[14] Winter, T.C., Ager, J.D., Nghiem, H.V., Hill, R.S., Harrison, S.D. and Freeny, P.C. (1996) Upper Gastrointestinal Tract and Abdomen: Water as an Orally Administered Contrast Agent for Helical CT. Radiology, 201, 365-370. 
http://dx.doi.org/10.1148/radiology.201.2.8888224

[15] Scanlon, M.H., Blumberg, M.L. and Ostrum, B.J. (1983) Computed Tomographic Recognition of Gastrointestinal Pathology. RadioGraphics, 3, 201-227. http://dx.doi.org/10.1148/radiographics.3.2.201

[16] Raptopoulos, V., Davis, M.A. and Smith, E.H. (1986) Imaging of the Bowel Wall: Computed Tomography and Fat Density Oral-Contrast Agent in an Animal Model. Investigative Radiology, 21, 847-850. http://dx.doi.org/10.1097/00004424-198611000-00003

[17] Garrett, P.R., Meshkov, S.L. and Perlmutter, G.S. (1984) Oral Contrast Agents in CT of the Abdomen. Radiology, 153, 545-546. http://dx.doi.org/10.1148/radiology.153.2.6484186

[18] Hebert, J.J., Taylor, A.J., Winter, T.C., Reichelderfer, M. and Weichert, J.P. (2006) Low-Attenuation Oral GI Contrast Agents in Abdominal-Pelvic Computed Tomography. Abdominal Imaging, 31, 48-53. http://dx.doi.org/10.1007/s00261-005-0350-4

[19] Mitka, M. (2007) Milk Shows Potential as CT Contrast Agent. Journal of the American Medical Association, $297,353$.

[20] Apisarnthanarak, P., Tiangpug, T., Pongpornsup, S., Janpanich, S. and Suwannasit, T. (2013) Comparison of Pasteurized Whole Milk, UHT Whole Milk, Water, and Diluted Iodine Contrast as Computed Tomographic Enteric Contrasts. Journal of the Medical Association of Thailand, 96, 467-476.

[21] Cohade, C., Osman, M., Nakamoto, Y., Marshall, L.T., Links, J.M., Fishman, E.K., et al. (2003) Initial Experience with Oral Contrast in PET/CT: Phantom and Clinical Studies. Journal of Nuclear Medicine, 44, 412-416.

[22] Thompson, S.E., Raptopoulos, V., Sheiman, R.L., McNicholas, M.M. and Prassopoulos, P. (1999) Abdominal Helical CT: Milk as a Low-Attenuation Oral Contrast Agent. Radiology, 211, 870-875. http://dx.doi.org/10.1148/radiology.211.3.r99jn25870

[23] Koo, C.W., Shah-Patel, L.R., Baer, J.W. and Frager, D.H. (2008) Cost-Effectiveness and Patient Tolerance of LowAttenuation Oral Contrast Material: Milk versus Volumen. American Journal of Roentgenology, 190, 1307-1313. http://dx.doi.org/10.2214/AJR.07.3193

[24] Lim, B.K., Bux, S.I., Rahmat, K., Lam, S.Y. and Liew, Y.W. (2012) Evaluation of Bowel Distension and Mural Visualisation Using Neutral Oral Contrast Agents for Multidetector-Row Computed Tomography. Singapore Medical Journal, 53, 732-736. 